Wartezeit dann nicht mehr als $30 \mathrm{Minu}-$ ten, außerdem gibt es besondere Sprechzeiten nur für Berufstätige.

Ein besonderer Standort im Netz ist das Gesundheitshaus Mirow an der Müritz. Diese von der Hausärztin Dr. Uta Arndt initiierte Einrichtung arbeitet so, wie es der Sachverständigenrat der Bundesregierung in seinem jüngsten Gutachten als Lösung für regionale Versorgungsprobleme vorschlägt nämlich als „lokales Gesundheitszentrum“.

\section{Mehrstufiges Konzept}

Dabei wird nach verschiedenen Versorgungsstufen unterschieden. Die erste, hausärztliche Versorgungsstufe wird im Gesundheitshaus durch zwei hausärztliche Praxen ausgefüllt. In der zweiten Ausbaustufe werden fachärztliche Zweigpraxen im Mirower Ärztehaus eingerichtet. Bereits vorhanden sind Sprechstunden für Gynäkologie, Orthopädie, Pulmologie und Schmerztherapie. Auch für HNO und Dermatologie werden noch Sprechstunden hinzukommen. Die beteiligten Fachärzte können vor Ort auf Behandlungsräume und Equipment zurückgreifen, so dass eine Erstinvestition entfällt. Zugleich profitieren sie von Netzvorteilen, etwa Verträgen zur Integrierten Versorgung.

In einer dritten Ausbaustufe kommen gerade telemedizinische Anwendungen, die Anbindung regionaler Kli- niken und Telekonsile mit den Schwerpunkten Pädiatrie und Dermatologie hinzu. Für die nähere Zukunft geplant sind Angebote zur Versorgung chronisch Kranker, etwa durch Förderung arztentlastender Versorgungsformen und durch die Qualifizierung von Pflegepersonal für die Schmerztherapie.

Die Rolle der TK ist dabei nicht nur die eines Vertragspartners, wie die TK-Landeschefin Manon Austenat-Wied im Gespräch betont: „Wir haben das Konzept mitentwickelt und es auf den Weg gebracht." Damit ist die Krankenkasse von vornherein daran beteiligt, welche Module im Praxisnetz für ihre Versicherten angeboten werden. „Grundsatz ist für uns, dass auch spezialärztliche Angebote in erreichbarer Nähe vorgehalten werden", betont AustenatWied.

Ein in vielen Bereichen ähnliches Konzept wie das Ärztehaus in Mirow hat die Gemeinde Woldegk, nur wenige Kilometer weiter an der Mecklenburgischen Seenplatte, umgesetzt - allerdings ohne die Anbindung an das Praxisnetz und von der Kommune initiiert (siehe MMW 3/2015, S. 8). Die TK schließt nicht aus, dass das Konzept aus Mirow Vorbild für andere Standorte sein könnte - angepasst an die jeweiligen lokalen Gegebenheiten.

Dirk Schnack

\title{
Schnelle Facharzttermin - im Nordosten längst etabliert
}

In der Debatte um schnellere Facharzttermine hatte die KV Mecklenburg-Vorpommern die Nase vorn. Bereits seit $\mathbf{2 0 0 8}$ wird im Nordosten ein Modell praktiziert, das den überweisenden Ärzten die Möglichkeit einräumt, Terminanfragen in A- und B-Kategorien einzuteilen.

Patienten mit hochakuten Erkrankungen erhalten eine A-Überweisung und werden innerhalb eines Werktages vom Facharzt übernommen. B-Termine werden an Patienten vergeben, bei denen sich bei einer laufenden Therapie eine Verschlechterung des Gesundheitszustands eingestellt hat. Diese Patienten erhalten innerhalb einer Woche einen Facharzttermin. Die überweisende Praxis schickt relevante Hintergrundinformationen mit, die übernehmende Praxis übermittelt im Anschluss Befunde und Behandlungsergebnisse an den überweisenden Arzt. Die KV sieht das Modell als Erfolg. Seit 2011 ist es auch Bestandteil von Vereinbarungen mit mehreren Krankenkassen - sowohl der überweisende als auch der spezialisierte Arzt erhalten ein Extra-Honorar.

\section{Aktuelle Urteile}

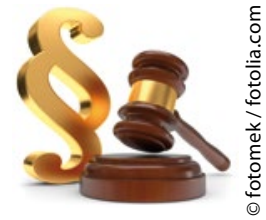

\section{Prüfgremien dürfen nicht jede Methode anwenden}

Bei der Wirtschaftlichkeitsprüfung dürfen die Prüfgremien nur solche Methoden anwenden, auf die sich Kassen und regionale KV vorher geeinigt haben. Das hat das Sozialgericht Berlin in einem noch nicht rechtskräftigen Urteil bestimmt. Ein Zahnarzt hatte gegen einen Regressbescheid geklagt, weil die Prüfer seine Kosten lediglich mit dem Durchschnitt der Fachgruppe verglichen hatten. Das ist eine einfache Methode, allerdings war sie nicht zwischen den Selbstverwaltungspartnern abgesprochen. Den Einwand der Prüfer, dass die vereinbarte Methode - eine Prüfung von mindestens 100 repräsentativen Einzelfällen mit Hochrechnung - zu aufwändig sein, ließen die Richter nicht gelten.

Der Medizinrechtler Torsten Münnch von der Kanzlei Dierks + Bohle, der den Kläger vertreten hatte, rät allen Ärzten, sich die Prüfvereinbarungen, die in jedem KV-Bezirk mit unterschiedlichen Inhalten existieren, genau anzusehen: „Gibt es da Lücken, steigen die Chancen, sich erfolgreich gegen Regresse zur Wehr zu setzen."

- chy

- SG Berlin, Az: S 79 KA 327/14

\section{Chlamydien-Infektion ist keine Berufskrankheit}

Eine Sonderschulerzieherin ist hinsichtlich der Chlamydia pneumoniae keiner Infektionsgefahr ausgesetzt, die in besonderem Maße über dem Risiko in der Gesamtbevölkerung liegt. Daher ist nach einem aktuellen, rechtskräftigen Urteil des Hessischen Landessozialgerichts eine Berufskrankheit nicht anzuerkennen.

Die Frau litt an Fieberschüben, Abgeschlagenheit und gehäuften Infekten der Atemwege. Sie machte geltend, dass im Rahmen ihrer beruflichen Tätigkeit eine ChlamydienInfektion eingetreten sei. Die Berufsgenossenschaft (BG) lehnte die Anerkennung als Berufskrankheit ab. Eine konkrete Infektionsquelle habe nicht nachgewiesen werden können. Jeder könne sich im täglichen Leben eine Chlamydien-Infektion zuziehen. Dem schlossen sich die Richter an. - maw

- LSGHessen, Az: L 3 U 54/11 